\title{
Robust PID controller design for nonlinear systems
}

\author{
Vojtech Veselý, Ladislav Körösi ${ }^{*}$
}

\begin{abstract}
In this paper the new approach to the design of robust PID controller for the case of nonlinear Lipschitz systems is proposed. The proposed method is based on the uncertain gain scheduling plant model and Bellman Lyapunov equation. The designed robust controller ensures parameter dependent quadratic stability and in the frame of $\mathrm{H}_{2}$ performance guaranteed cost. Examples show the effectiveness of the proposed method.
\end{abstract}

K e y w o r d s: robust controller; Lipschitz systems; gain scheduled controller, Bellman Lyapunov equation

\section{Introduction}

PID controllers have found large number of practical applications, in the more than eight decades since Ziegler and Nichols proposed their first PID controller tuning method. Tuning rules of PID controller parameters can be divided into the following groups based on:

- measured step response.

- a measure of critical (ultimate) points of closed- control loop system.

- the mathematical plant model and model uncertainties analytical approach.

Due to [7], there are more than 184 tuning rules for PI and 258 tuning rules for PID controllers. Most of them are suited for a particular problem or give the specified closed-loop performance. In the paper [12] the PID tuning rules for specified performance for stable or not stable systems have been proposed. The paper [8] is devoted to design of the PID controller gains using gain margin methods to compensate the instability of closed loop system which may induced by time delays for the network control system. Robust PID controller parameters design for mechanical actuators using Kharitonovs theorem is given in [10]. In the frequency domain with uncertainties [4] linear programming is used for the design of robust PID controllers. Robust PID controller design for linear MIMO system is given $[1,3,5,15]$. For nonlinear Lipschitz systems, there are two basic methods one could use the Lyapunov function or the linearization and multimodel approach. The latest approaches lead to the use of switched controller design [13] or linear parameter varying system (LPV). In this paper, we will use the idea to transform the nonlinear system to the linear parameter varying (LPV) system [6], and for LPV system develop the new design procedure for the design of robust PID controller. In this paper two design procedures have been developed. The first method is less conservativeness than the second ones, but the computation loads are greater for the first method.

After the problem formulation, transformation of nonlinear systems to LPV model, some preliminary results are given. A sufficient robust stability conditions in the form of BMI for proposed robust PID controller design procedures are introduced, and obtained results are illustrated on two examples. Hereafter the following notational conditions will be adopted. Given a symmetric matrix $P=P^{\top} \in R^{n \times m}$ the inequality $P>0$ means the matrix positive definiteness. $I$ denotes the identity matrix.

\section{Problem statement and preliminaries}

Consider the uncertain nonlinear Lipschitz systems

$$
\dot{z}=f(z, v, w), \bar{y}=h(z)
$$

where $z \in R^{\bar{n}}$ is the state, the input, $v \in R^{m}$, the output $\bar{y} \in R^{\bar{l}}$ and exogenous input $w \in R^{k}$, which captures the parametric dependence of the plant model on exogenous input. Assume that the nonlinear function $f($. is locally Lipschitz, that is for each state vector $z_{0} \in R^{\bar{n}}$ one can linearize the nonlinear function $f($.$) . Let the$ number of linearized points of system be $\bar{p}$. Transform plant model (1) to the linear parameter varying system using the following steps.

1 - Choose the number of gain scheduled variables $\theta, p=$ $\bar{p}-1$ and their upper $(\bar{\theta})$ and lower bound $(\underline{\theta})$ as a function of exogenous input $w$.

2 - For robust controller design choose $r$ uncertain parameters of the plant (1) which are ranges within given two values.

3 - At each linearized point $\bar{j}=1,2, \ldots, \bar{p}$ and uncertain parameters $i=1,2, \ldots, 2^{r}$ one builds a linearized

* Institute of Robotics and Cybernetics, Slovak University of Technology in Bratislava, Slovakia, vojtech.vesely@stuba.sk 
model of the plant (1)

$$
\begin{aligned}
& \overline{\dot{x}}=\bar{A}_{i j} \bar{x}+\bar{B}_{i j} u \\
& y_{s}=\bar{C} \bar{x}
\end{aligned}
$$

where, $\bar{x}=z-z_{e}, u=v-v_{e}, y_{s}=\bar{y}-y_{e},\left(z_{e}, v_{e}, \bar{y}_{e}\right)$ define the equilibrium points of the nonlinear system (1) at each linearized point for the case of different combinations of $\theta$. For each $i$ we transform (2) to the gain scheduling time varying model using simple equality.

$$
\begin{aligned}
{\left[\begin{array}{c}
\bar{A}_{i 1} \\
\bar{A}_{i 2} \\
\vdots \\
\bar{A}_{i \bar{p}}
\end{array}\right]=} & {\left[\begin{array}{cccc}
I \underline{Q}_{1} & \cdots & I \underline{Q}_{p} & \\
\vdots & \vdots & \vdots & \vdots \\
I & I \bar{Q}_{1} & \cdots & I \bar{Q}_{p}
\end{array}\right]\left[\begin{array}{c}
\bar{A}_{0 i} \\
\bar{A}_{1 i} \\
\vdots \\
\bar{A}_{p i}
\end{array}\right], }
\end{aligned}
$$

From which one obtains

$$
\begin{aligned}
& \bar{A}_{i}(\theta)=\bar{A}_{0 i}+\sum_{j=1}^{p} \bar{A}_{i j} \theta_{j} \in R^{\bar{n} \times \bar{m}} \\
& \bar{B}_{i}(\theta)=\bar{B}_{0 i}+\sum_{j=1}^{p} \bar{B}_{i j} \theta_{j} \in R^{\bar{n} \times \bar{m}}
\end{aligned}
$$

Due to uncertainties the obtained system matrices are in the polytope with $K=2^{r}$ vertices which can be formally defined as

$$
\begin{gathered}
\Omega=\{\bar{A}(\xi, \theta), \bar{B}(\xi, \theta)\}=\sum_{i=1}^{K}\left(\bar{A}_{i}(\theta), \bar{B}_{i}(\theta)\right) \xi_{i} \\
\sum_{i=1}^{K} \xi_{i}=1, \quad \xi_{i} \geqslant 0, \quad \xi_{i} \in \Omega_{\xi}, \quad \dot{\xi}_{i} \in \Omega_{t \xi}
\end{gathered}
$$

where, $\xi_{i}, i=1,2, \ldots K$ are constant or possible time varying, but unknown parameters satisfying (4), $\theta \in R^{p}$ is a vector of known constant or time varying gain scheduled parameters. We assume that lower, upper bounds and their rates are available, that is

$$
\begin{aligned}
& \theta \in \Omega_{\theta}=\left\{\theta \in R^{p}: \theta_{j} \in\left\langle\underline{\theta}_{j}, \bar{\theta}_{j}\right\rangle\right\} \\
& \dot{\theta} \in \Omega_{\theta}=\left\{\dot{\theta} \in R^{p}: \dot{\theta}_{j} \in\left\langle\underline{\dot{\theta}}_{j}, \dot{\bar{\theta}}_{j}\right\rangle\right\}
\end{aligned}
$$

For more detail how to obtain the gain scheduled plant model, see the excellent surveys [6]. The following problem is studied in this paper. Design a robust static output feedback PID controller with control algorithm

$$
u(t)=K_{p} y_{s}+K_{i} \int_{\tau=0}^{t} y_{s}(\tau) \mathrm{d} \tau+K_{d} \dot{y}_{s}(t)
$$

such that the controller (6) with (9) ensures closed loop parameter dependent quadratic stability and guaranteed cost in the frame of $\mathrm{H}_{2}$.

The plant model (4) can be rewritten as in (7)

$$
\dot{\bar{x}}=\bar{A}(\xi, \theta) \bar{x}+\bar{B}(\xi, \theta) u, \quad y_{s}=\bar{C} \bar{x}
$$

Due to the integral part of the PID controller, lets introduce the new system output as $e=\int_{0}^{t} y_{s}(\tau) d t \in R^{\bar{l}}$. System (7) is

$$
\dot{x}=\left[\begin{array}{c}
\dot{\bar{x}} \\
\dot{e}
\end{array}\right]=\left[\begin{array}{cc}
\bar{A}(\xi, \theta) & 0 \\
\bar{C} & 0
\end{array}\right]\left[\begin{array}{c}
\bar{x} \\
e
\end{array}\right]+\left[\begin{array}{c}
\bar{B}(\xi, \theta) \\
0
\end{array}\right] u
$$

Output

$$
\begin{aligned}
& y=\left[\begin{array}{c}
y_{s} \\
e
\end{array}\right]=C x, \\
& C=\left[\begin{array}{cc}
\bar{C} & 0 \\
0 & I_{e}
\end{array}\right] \in R^{(2 \bar{l}) \times(\bar{n}+\bar{l})} \in R^{l \times n}
\end{aligned}
$$

or

$$
\dot{x}=A(\xi, \theta) x+B(\xi, \theta) u, \quad y=C x
$$

Due to the augmented plant model (8) control algorithm (6) is in the form

$$
u(t)=K_{p} C_{p} x+K_{i} C_{i} x+K_{d} C_{d} \dot{x}
$$

where

$$
\begin{aligned}
& C_{p}=\left[\begin{array}{ll}
\bar{C} & 0
\end{array}\right] \in R^{\bar{l} \times n}, \\
& C_{i}=\left[\begin{array}{ll}
0 & I_{\bar{e}}
\end{array}\right] \in R^{\bar{l} \times n} .
\end{aligned}
$$

To assess performance quality, the following cost function is used within $\mathrm{H}_{2}$ frame

$$
J_{c}=\int_{0}^{\infty} J(x, u, \dot{x}) \mathrm{d} t
$$

where $J(x, u, \dot{x})=x^{\top} Q x+u^{\top} R u+\dot{x}^{\top} S \dot{x}$ and $(Q, S) \in$ $R^{n \times n}$ are positive definite (semidefinite) matrices, and $R \in R^{m \times m}$ is positive definite matrix. Lemma 1. Consider the uncertain plant model (9) with control algorithm (10). Control algorithm (10) is the guaranteed cost control law for the closed loop system if and only if a Lyapunov function $V(x, \theta, \xi)$ exists such that the following inequality holds

$$
B_{e}=\frac{d V(\cdot)}{d t}+J(x, u, \dot{x}) \leq-\varepsilon x^{\top} x, \varepsilon \rightarrow 0, \varepsilon \geq 0
$$

The equation (12) is known as the Bellman-Lyapunov equation, and function, which satisfies (12), is the Lyapunov function. For the particular structure of the Lyapunov function, the obtained robust controller design procedure may reduce from if and only if to if. 


\section{Robust controller design}

This part of the paper is devoted to develop a new robust PID controller design procedure for the case of gain scheduled plant model and polytope uncertainty. The obtained PID controller ensures to closed loop system parameter dependent quadratic stability for the all $\theta \in \Omega_{\theta}$, ,$\xi \in \Omega_{\xi}$ and $\dot{\xi} \in \Omega_{t \xi}$. The two controller design procedure is obtained. Let in (12) the Lyapunov function be given as $V(x, \theta, \xi)=x^{\top} P(\xi, \theta) x$ where

$$
P(\xi, \theta)=P_{0}(\xi)+\sum_{j=1}^{p} P_{j}(\xi) \theta_{j}=\sum_{i=1}^{K}\left(P_{i 0}+\sum_{j=1}^{p} P_{i j} \theta_{j}\right) \xi_{i}
$$

\subsection{First controller design procedure}

The first time derivative of the Lyapunov function (13) is as follows

$$
\frac{d V}{d t}=\left[\dot{x}^{\top} x^{\top} u^{\top}\right]\left[\begin{array}{ccc}
0 & P(\theta, \xi) & 0 \\
P(\theta, \xi) & P(\dot{\theta}, \dot{\xi}) & 0 \\
0 & 0 & 0
\end{array}\right]\left[\begin{array}{l}
\dot{x} \\
x \\
u
\end{array}\right]
$$

where

$$
\begin{gathered}
P(\dot{\xi}, \dot{\theta})=\left(\sum_{i=1}^{K}\left(P_{i 0}+\sum_{j=1}^{p} P_{i j} \theta_{j}\right) \xi_{i}\right)^{\prime}= \\
=\sum_{i=1}^{K}\left(P_{d i 0}+\sum_{j=1}^{n} P_{d i j} \theta_{j}\right) \xi_{i} \\
P_{d i 0}=\sum_{i=1}^{K} P_{i 0} \dot{\xi}_{i}+\sum_{j=1}^{p} P_{i j} \dot{Q}_{j}, \quad P_{d i j}=\sum_{i=1}^{K} P_{i j} \dot{\xi}_{i}
\end{gathered}
$$

To ensure less conservativeness results and split system matrices from Lyapunov matrix introduce the auxiliary matrices

$$
\begin{aligned}
& N_{i} \in R^{n \times n}, \quad i=1,2, \quad N_{3} \in R^{n \times m}, \\
& N_{i} \in R^{m \times n}, \quad i=4,5, \quad N_{6} \in R^{m \times m}
\end{aligned}
$$

as follows

$$
\begin{aligned}
& 2\left(N_{1} \dot{x}+N_{2} x+N_{3} u\right)^{\top}(\dot{x}-A(\xi, \theta) x-B(\xi, \theta) u)=0 \\
& 2\left(N_{4} \dot{x}+N_{5} x+N_{6} u\right)^{\top}\left(u-K_{p} C_{p} x-K_{i} C_{i} x-K_{d} C_{d} \dot{x}\right) \\
& =0
\end{aligned}
$$

From (16a) one obtains

$$
\begin{aligned}
{\left[\dot{x}^{\top} x^{\top} u^{\top}\right]\left[\begin{array}{cc}
N_{1}^{\top}+N_{1} & -N_{1}^{\top} A(\cdot)+N_{2} \\
N_{2}^{\top}-A^{\top}(\cdot) N_{1} & -N_{2}^{\top} A(\cdot)-A^{\top}(\cdot) N_{2} \\
N_{3}^{\top}-B^{\top}(\cdot) N_{1} & -N_{3}^{\top} A(\cdot)-B^{\top}(\cdot) N_{2} \\
& \\
N_{3}-N_{1}^{\top} B(\cdot) \\
-N_{2}^{\top} B(\cdot)-A^{\top}(\cdot) N_{3} \\
-N_{3}^{\top} B(\cdot)-B^{\top}(\cdot) N_{3}
\end{array}\right]\left[\begin{array}{c}
\dot{x} \\
x \\
u
\end{array}\right]=0 }
\end{aligned}
$$

and from $(16 \mathrm{~b})$

$$
\begin{aligned}
& {\left[\dot{x}^{\top} x^{\top} u^{\top}\right]\left[\begin{array}{l}
2 N_{4}^{\top} \\
2 N_{5}^{\top} \\
2 N_{6}^{\top}
\end{array}\right]\left[-K_{d} C_{d}-K_{c} I\right]\left[\begin{array}{l}
\dot{x} \\
x \\
u
\end{array}\right]=0} \\
& K_{c}=K_{p} C_{p}+K_{i} C_{i}
\end{aligned}
$$

Substituting (11), (14), (16) and (17) to (12) after manipulation we obtain the Bellman-Lyapunov function in the form

$$
B_{e}=\left[\dot{x}^{\top} x^{\top} u^{\top}\right] W(\xi, \theta)\left[\dot{x}^{\top} x^{\top} u^{\top}\right]^{\top} \leq 0,
$$

where:

$$
\begin{aligned}
& W(\xi, \theta)=\left\{w_{i j}(\xi, \theta)\right\} \\
& w_{11}(\xi, \theta)=N_{1}^{\top}+N_{1}-N_{4}^{\top} K_{d} C_{d} \\
& -C_{d}^{\top} K_{d}^{\top} N_{4}+S \\
& w_{12}(\xi, \theta)=P(\xi, \theta)-N_{1}^{\top} A(\xi, \theta)+N_{2}-N_{4}^{\top} K_{c} \\
& -C_{d}^{\top} K_{d}^{\top} N_{5} \\
& w_{13}(\xi, \theta)=N_{3}-N_{1}^{\top} B(\xi, \theta)+N_{4}^{\top}-C_{d}^{\top} K_{d}^{\top} N_{6} \\
& w_{22}(\xi, \theta)=-N_{2}^{\top} A(\xi, \theta)-A(\xi, \theta)^{\top}-N_{5}^{\top} K_{c} \\
& -K_{c}^{\top} N_{5}+P(\dot{\xi}, \dot{\theta})+Q \\
& w_{23}(\xi, \theta)=-N_{2}^{\top}(\xi, \theta)-A^{\top}(\xi, \theta) N_{3}+N_{5}^{\top}-K_{c}^{\top} N_{6} \\
& w_{33}(\xi, \theta)=-N_{3}^{\top} B(\xi, \theta)-B(\xi, \theta) N_{3}+N_{6}^{\top}+R
\end{aligned}
$$

Correspondingly, all elements of matrix $W(\xi, \theta)$ are convex with respect to uncertainly $\xi$ and $\theta$, one can split the matrix $W(\xi, \theta)$ as follows.

$$
W_{k e}(\xi, \theta)=\sum_{k=1}^{K}\left(W_{k l i 0}+\sum_{j=1}^{p} W_{k e i j} \theta_{j}\right) \xi_{i}
$$

or robust stability condition is given as

$$
W(\xi, \theta)=\sum_{k=1}^{K}\left(W_{i 0}+\sum_{j=1}^{p} W_{i j} \theta_{j}\right) \xi_{i} \leq 0
$$

where:

$$
\begin{aligned}
& w_{11 i 0}=N_{1}^{\top}+N_{1}-N_{4}^{\top} K_{d} C_{d}-C_{d}^{\top} K_{d}^{\top} N_{4}+S, \\
& w_{11 i j}=0 \\
& w_{12 i 0}=P_{i 0}-N_{1}^{\top} A_{i 0}+N_{2}-N_{4}^{\top} K_{c}-C_{d}^{\top} K_{d}^{\top} N_{5} \\
& w_{12 i j}=P_{i j}-N_{1}^{\top} A_{i j} \\
& w_{13 i 0}=N_{3}-N_{1}^{\top} N B_{i 0}+N_{4}^{\top}-C_{d}^{\top} K_{d}^{\top} N_{6} \\
& w_{13 i j}=-N_{1}^{\top} B_{i j} \\
& w_{22 i 0}=-N_{2}^{\top} A_{i 0}-A_{i 0}^{\top} N_{2}-N_{5}^{\top} K_{c}-K_{c}^{\top} N_{5}+ \\
& Q+P_{d i 0}
\end{aligned}
$$




$$
\begin{aligned}
& w_{22 i j}=-N_{2}^{\top} A_{i j}-A_{i j}^{\top} N_{2}+P_{d i j} \\
& w_{23 i 0}=-N_{2}^{\top} B_{i 0}-A_{i 0}^{\top} N_{3}+N_{5}^{\top}-K_{c}^{\top} N_{6} \\
& w_{23 i j}=-N_{2}^{\top} B_{i j}-A_{i j}^{\top} N_{3} \\
& w_{33 i 0}=-N_{3}^{\top} B_{i 0}-B_{i 0}^{\top} N_{3}+N_{6}^{\top}+N_{6}+R \\
& w_{33 i j}=-N_{3}^{\top} B_{i j}-B_{i j}^{\top} N_{3}
\end{aligned}
$$

The obtained first main results are summarized to the following theorem.

\section{Theorem 1}

The uncertain gain scheduled system (9) with controller (10) is robust PDQS with guaranteed cost, if there exists a positive definite Lyapunov matrix $P(\xi, \theta) \in$ $R^{n \times n}$, matrices $N_{k}, k=1,2, . ., 6$, positive definite matrix $R$ and positive definite (semidefinite) matrices $Q, S$, such that inequality (20) holds for all $\theta \in \Omega_{0}, \dot{\theta} \in \Omega_{t}, \dot{\xi} \in$ $\Omega_{\xi t}$. Proof. Due to the concrete Lyapunov function (13), the sufficient robust stability conditions follow (12), (13), (14) and (18). Note that (20) are convex with respect to the gain scheduled parameters $\theta$ and uncertainty $\xi$. Inequalities (20) hold if and only if they are negative for all $i=1,2, \ldots K$ and corners of gain scheduled parameters $\theta_{j} j=1,2, \ldots, p$ and their rates. As the rates of $\theta$ and $\xi$ are in the diagonal of matrix $W(\xi, \theta)$, we can reduce the computation load using the inequalities.

$$
\begin{aligned}
& \sum_{i=1}^{K} P_{i 0} \dot{\xi}_{i} \leq \sum_{i=1}^{K} P_{i 0} \overline{\dot{\xi}}_{i} \\
& \sum_{j=1}^{p} P_{i j} \dot{\theta}_{j} \leq \sum_{j=1}^{p} P_{i j} \overline{\dot{\theta}}_{j}
\end{aligned}
$$

and $\sum_{i=1}^{K} P_{i j} \dot{\xi}_{i} \leq \sum_{i=1}^{K} P_{i j} \overline{\dot{\xi}}_{i}$ assuming that: $P_{i j}>0, i=$ $1,2 . ., K, j=01,2, . ., p$

\subsection{Second robust controller design}

Closed loop system for uncertain plant model (9) and controller (10) one obtains as

$$
\dot{x}=A(\xi, \theta) x+B(\xi, \theta)\left(K_{c} x+K_{d} C_{d} \dot{x}\right)
$$

or

$$
M(\xi, \theta) \dot{x}=A_{c}(\xi, \theta) x
$$

where

$$
\begin{array}{r}
M(\xi, \theta)=\left[I-B(\xi, \theta) K_{d} C_{d}\right]= \\
=\sum_{i=1}^{k}\left(M_{i 0}+\sum_{j=1}^{p} M_{i j} \theta_{j}\right) \xi_{i} \\
A_{c}(\xi, \theta)=A(\xi, \theta)+B(\xi, \theta) K_{c}= \\
=\sum_{i=1}^{k}\left(A_{c i 0}+\sum_{j=1}^{p} A_{c i j} \theta_{j}\right) \xi_{i}
\end{array}
$$

$$
\begin{gathered}
M_{i 0}=I-B_{i 0} K_{d} C_{d}, \quad M_{i j}=-B_{i j} K_{d} C_{d} \\
A_{c i 0}=A_{i 0}+B_{i 0} K_{c}, \quad A_{c i j}=A_{i j}+B_{i j} K_{c}
\end{gathered}
$$

For obtaining the convex robust stability condition with respect to gain scheduled and uncertainty parameters introduce two auxiliary matrices $M_{1}, M_{2} \in R^{n \times n}$ as

$$
\left[\begin{array}{ll}
\dot{x}^{\top} & x^{\top}
\end{array}\right]\left[\begin{array}{l}
2 M_{1}^{\top} \\
2 M_{2}^{\top}
\end{array}\right]\left[M(\xi, \theta)-A_{c}\right]\left[\begin{array}{l}
\dot{x} \\
x
\end{array}\right]=0
$$

Quadratic performance (11) one can rewrite as

$$
\begin{aligned}
& J=x^{\top} Q x+\dot{x}^{\top} S \dot{x}+u^{\top} R u \Rightarrow \\
& {\left[\begin{array}{ll}
\dot{x}^{\top} & x^{\top}
\end{array}\right]\left[\begin{array}{cc}
C_{d}^{\top} K_{d}^{\top} R K_{d} C_{d}+S & C_{d}^{\top} K_{d}^{\top} R K_{c} \\
K_{c}^{\top} R K_{c} C_{d} & K_{c}^{\top} R K_{c}+Q
\end{array}\right]\left[\begin{array}{c}
\dot{x} \\
x
\end{array}\right]}
\end{aligned}
$$

Summarizing (14), (24), (25) and substitute the obtained results to (12) one obtains

$$
B_{e}=v^{\top} V(\xi, \theta) v \leq 0
$$

where

$$
V(\xi, \theta)=\left\{v_{i j}(\xi, \theta)\right\}_{2 \times 2}=\sum_{i=1}^{K}\left(v_{i 0}+\sum_{j=1}^{p} v_{i j} \theta_{j}\right) \xi_{i}
$$

and

$$
\begin{aligned}
& v_{11 i 0}=M_{1}^{\top} M_{i 0}+M_{i 0}^{\top} M_{1}+C_{d}^{\top} K_{d}^{\top} R K_{d} C_{d}+S \\
& v_{11 i j}=M_{1}^{\top} M_{i j}+M_{i j}^{\top} M_{1} \\
& v_{12 i 0}=-M_{1}^{\top} A_{c i 0}+M_{i 0}^{\top} M_{2}+P_{i 0}+C_{d}^{\top} K_{d}^{\top} R K_{c} \\
& v_{12 i j}=-M_{1}^{\top} A_{c i j}+M_{i j}^{\top} M_{2}+P_{i j} \\
& v_{22 i 0}=-M_{2}^{\top} A_{c i 0}-A_{c i 0}^{\top} M_{2}+K_{c}^{\top} R K_{c}+Q+P_{d i 0} \\
& v_{22 i j}=-M_{2}^{\top} A_{c i j}-A_{c i j}^{\top} M_{2}+P_{d i j}
\end{aligned}
$$

The obtained results are summarized in the following theorem

\section{Theorem 2}

The uncertain gain scheduled system (9) with the controller (10) is robust PDQS with guaranteed cost if there exists a positive definite matrix $P(\xi, \theta)$, matrices $M_{1}, M_{2}$ and positive definite $R$, and positive definite (semidefinite) $Q, R$ such that inequality (26) holds for all $\theta \in \Omega_{0}, \dot{\theta} \in \Omega_{t}, \quad \dot{\xi} \in \Omega_{\xi t}$. Note that (26) may be more conservative than (20). For the case of an uncertain linear system, the robust stability conditions (20), (26) reduces to

$$
W_{i 0} \leq 0, \quad V_{i 0} \leq 0, \quad i=1,2, \ldots K
$$

The latest condition has been obtained for the case of robust controller design by [8], and for the case of PIDPSD robust controller design with guaranteed cost in [11]. 


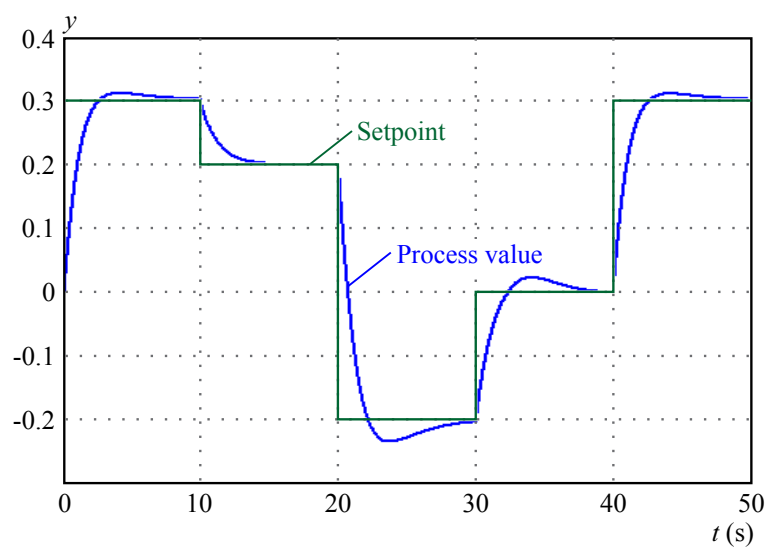

Fig. 1. Time responses of the controlled system (1. method)

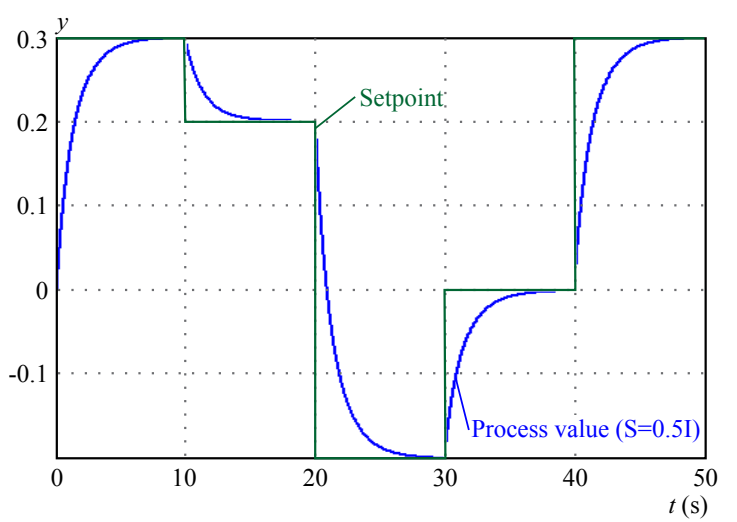

Fig. 3. Time responses of the controlled system (2nd method)

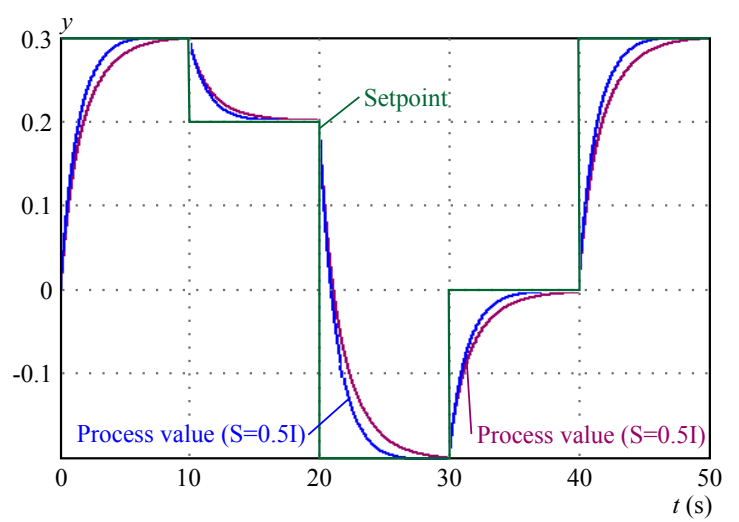

Fig. 5. Time responses of the controlled system (2nd method) detailed zoom

Example 1 - The first example is borrowed from [2]. It is a simple nonlinear system which has an unstable zero equilibrium point.

$$
\begin{aligned}
& \dot{x}=-x|x|+u \\
& y=x \\
& -0.5 \leq u \leq 0.5 \\
& -0.5 \leq y \leq 0.5
\end{aligned}
$$

One can transformed above system it into the following form

$$
\dot{x}=-\left(a_{0}+a_{1} \theta\right) x+b u, \quad y=C x
$$

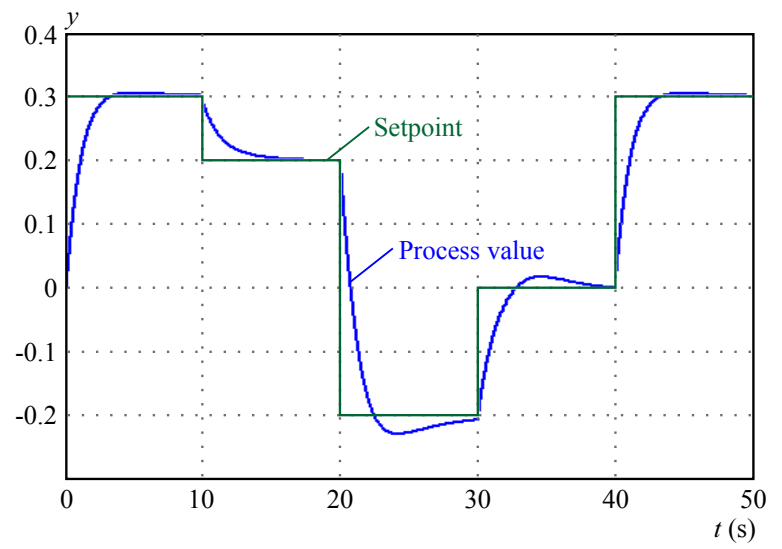

Fig. 2. Time responses of the controlled system (1. method) - zoom

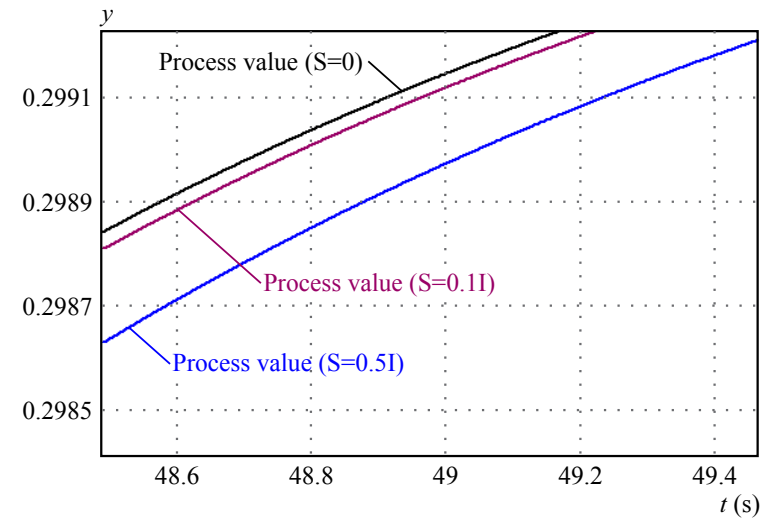

Fig. 4. Time responses of the controlled system (2nd method) zoom

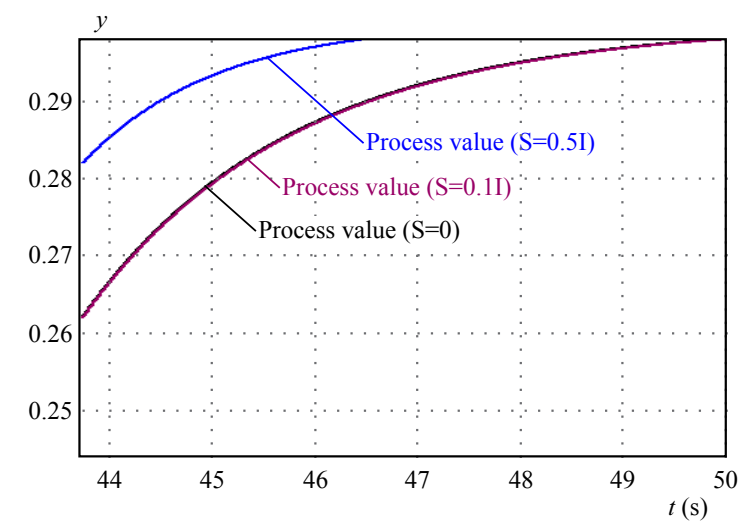

Fig. 6. Time responses of the controlled system (2nd method) zoom

where $b \in\langle 1,1.5\rangle, C=1, a_{1}=-0.25, a_{0}=0.25$ and $\theta \in\langle-1,1\rangle$. For PI controller introduce the new state $e=\int_{0}^{t} y d t$, we obtain

$$
\begin{aligned}
& {\left[\begin{array}{c}
\dot{x} \\
\dot{e}
\end{array}\right]=\left[\begin{array}{cc}
a_{0}+a_{1} \theta & 0 \\
1 & 0
\end{array}\right]\left[\begin{array}{l}
x \\
e
\end{array}\right]+\left[\begin{array}{l}
b \\
0
\end{array}\right] u=} \\
& {\left[\begin{array}{cc}
a_{0} & 0 \\
1 & 0
\end{array}\right]\left[\begin{array}{l}
x \\
e
\end{array}\right]+\left[\begin{array}{cc}
a_{1} & 0 \\
0 & 0
\end{array}\right]\left[\begin{array}{l}
x \\
e
\end{array}\right] \theta+\left[\begin{array}{l}
b \\
u
\end{array}\right]}
\end{aligned}
$$

The aim is to design a robust PID controller which will guarantee PDQS, using in the paper the proposed meth- 


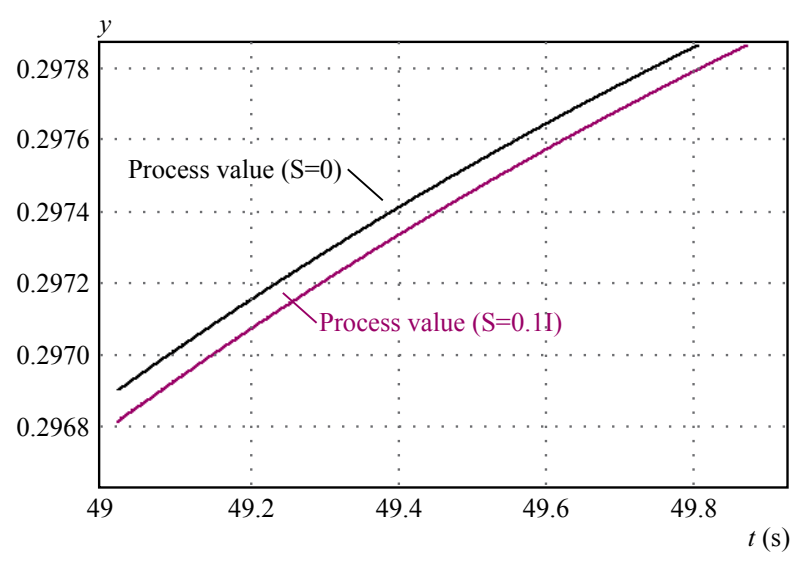

Fig. 7. Time responses of the controlled system 2 nd method detailed zoom

ods. For the following parameters

$$
\begin{aligned}
& 0<P_{i j}<r_{0} I, r_{0}=100, \overline{\dot{\theta}}=0.5 / s, \overline{\dot{\xi}}=0.2 / s, \\
& R=I, Q=0.001 I, S=0
\end{aligned}
$$

one obtaines PID controllers $R(s)=\alpha+\frac{\beta}{s}+\gamma s$, as shown in Tab. 1

Table 1. Example 1 controller parameters and eigenvalues

\begin{tabular}{ccccc}
\hline Method & $\alpha$ & $\beta$ & $\gamma$ & Max. eigen. cl. \\
\hline 1 & -1.0473 & -0.4713 & 0.1577 & -0.4733 \\
2 & -0.9876 & -0.3765 & 0.1532 & $0.4355^{*}$ \\
\hline * a more conservative result
\end{tabular}

The simulation results for different $\mathrm{S}$ are shown in Fig. 3 till Fig. 7. The results for the first model are almost similar, there is only a small influence of the $\mathrm{S}$ on the time response (detailed zoom is in Fig. 4). Higher impact on the dynamics regarding of the value of $\mathrm{S}$ can be seen in Fig. 5 to Fig. 7. The higher value of $\mathrm{S}$ increases the rate of the model output which leads to faster dynamics of the process.

Example 2 - In the second example we have used a single nonlinear system

$$
\begin{aligned}
& \dot{x}=-a \sin x+b u, \text { where: } a \in\langle 0.8,1\rangle, \\
& \text { when } a=0.8, \text { then } b=1 \\
& \text { and when } a=1, b=-0.5
\end{aligned}
$$

One can linearize the above model in the three working points $x_{0}=\{0, \pi / 4, \pi / 2\}$. For PI robust controller design the system state space needs to be augmented, finally for matrices $A(\xi, \theta), B(\xi, \theta)$, one obtains:

$$
\begin{aligned}
& A(\xi, \theta)=\left\{\left[\begin{array}{cc}
-0.4 & 0 \\
1 & 0
\end{array}\right]+\left[\begin{array}{cc}
0.117 & 0 \\
0 & 0
\end{array}\right] \theta_{1}+\right. \\
& \left.\left[\begin{array}{cc}
-0.28 & 0 \\
0 & 0
\end{array}\right] \theta_{2}\right\} \xi_{1}+\left\{\left[\begin{array}{cc}
-0.5 & 0 \\
1 & 0
\end{array}\right]+\right. \\
& \left.\left[\begin{array}{cc}
0.14645 & 0 \\
0 & 0
\end{array}\right] \theta_{1}+\left[\begin{array}{cc}
-0.35355 & 0 \\
0 & 0
\end{array}\right] \theta_{2}\right\} \xi_{2}
\end{aligned}
$$

The aim is to design a robust PID controller, and in an experimental way show how the matrix $\mathrm{S}$ influences the dynamic behavior of the closed loop system. Under the parameters $0<P_{i j}<r_{0} I, r_{0}=100, \overline{\dot{\theta}}_{i}=0.5, i=$ $1,2, \overline{\dot{\xi}}=0.1, R=I, Q=0.0001 I, S=0$.

Obtained PID controllers are in Tab. 2

Table 2. Example 2 controller parameters and eigenvalues

\begin{tabular}{ccccc}
\hline Method & $\alpha$ & $\beta$ & $\gamma$ & Max. eigen. cl. \\
\hline 1 & -0.8633 & -0.5823 & 0.0870 & -0.487 \\
2 & -0.7114 & -0.4545 & 0.0738 & -0.4443 \\
\hline \multicolumn{5}{c}{ Case $S=0.1 I$} \\
\hline 1 & -0.7146 & -0.5822 & 0.0873 & -0.4879 \\
2 & -0.7114 & -0.4538 & 0.0738 & 0.074 \\
\hline \multicolumn{5}{c}{ Case $S=0.5 I$} \\
\hline 1 & -0.8753 & 0.5787 & 0.0860 & -0.4899 \\
2 & -0.8351 & -0.5999 & 0.01031 & -0.4837 \\
\hline
\end{tabular}

\section{Conclusion}

The paper devoted to the robust controller design for the nonlinear Lipschitz system. Designed controller guarantees closed loop robust stability and PDQS. A new method of PID controller design procedure based on the gain scheduled plant LPV model. The obtained results are in the form of BMI. In the paper two controller design procedures were proposed, where the first proposed method is less conservative than the second. The obtained results were summarized in the theorems and verified using two examples, which show the effectiveness of the proposed method. Numerical solution has been carried out by MATLAB 7.5 using YALMIP with solver PENBMI21.

\section{Acknowledgment}

The work has been supported by the Slovak Scientific Grant Agency, Grant No. 1/0475/16.

\section{REFERENCES}

[1] M. Ge, M. -S. Chiu and Q. -G. Wang, "Robust PID controller design via the LMI approach", Journal of Process Control, vol. 12, pp. 3-13, 2002.

[2] M. F. Hassan and M. Zribi, "An observer-based controller for nonlinear systems: A gain scheduling approach", Applied mathematics and computation, vol. 237, pp. 695-711, 2014.

[3] Z. Hu, Y. He and W. Li, "Robust PID Controller Design for Multivariable Processes", Proceedings of the 2008 Fourth International Conference on Natural Computation, vol. 04, pp. 427-431, 2008. 
[4] A. Karimi, M. Kunze and R. Longchamp, "Robust PID controller design by linear programming", American Control Conference, 14-16 June 2006.

[5] A. Kozáková, V. Veselý and J. Osuský, "A new Nyquist-based technique for tuning robust decentralized controllers", Kybernetika, vol. 45, no. 1, pp. 63-83, 2009.

[6] D. J. Leith and W. E. Leithead, "Survey of gain-scheduling analysis and design", vol. 73, no. 11, pp. 1001-1025, 2000.

[7] A. O'Dwyer and :, "A Summary of PI and PID Controller Tuning Rules for Processes with Time Delay", IFAC Workshop PID'00, Terrasa, Part 1: pp. 175-180, Part 2: pp. 242-247, 2000.

[8] D. Pansari, D. Ch,rakar, B. Tim,e, "A Robust PID Controller Design For Network Control System", vol. 1, no. 7, pp. 1-5, September 2012.

[9] D. Peaucelle, D. Arzelier, O. Bachelier and J. Bernussou, "A new robust D-stability condition for real convex polytopic uncertainty", Systems and Control Letters, vol. 40, no. 1, pp. 21-30, 2000.

[10] R. Salloum, B. Moaveni and M. R. Arvan, "Robust PID Controller Design for a Real Electromechanical Actuator", Acta Polytechnica Hungarica, vol. 11, no. 5, 2014.

[11] V. Veselý and D. Rosinový, "Robust PID-PSD Controller Design: BMI Approach", Asian Journal of Control, vol. 15, no. 2, pp. 469-428, 2013.

[12] V. Veselý, "Easy Tuning of PID Controller", J. Electrical Engineering, vol. 54, no. 5-6, pp. 136-139, 2003.

[13] V. Veselý and A. Ilka, "Novel approach to switched controller design for linear continuous-time systems", Asian Journal of Control, vol. 18, no. 4, pp. 1365-1375, 2016.

[14] M. Vítečková, A. Víteček and L. Smutný, "Simple PI and PID Controllers Tuning for Monotone Self-Regulating Plants", IFAC Workshop PID'00, Terrasa, pp. 289-294, 2000.
15] J. Wang, S. Ma and C. Zhang, "Stability analysis and stabilization for nonlinear continuous-time descriptor semi-Markov jump systems", Applied Mathematics and Computation, vol. 279, pp. 90-102, 2016.

16] J. G. Ziegler and N. B. Nichols, "Optimum settings for automatic controllers", Transactions of the ASME. vol. 64, pp. 759768,1942 .

Received 14 November 2017

Vojtech Veselý was born in Velké Kapušany, Slovakia in 1940. He received MSc degree in Electrical Engineering from the Leningrad Electrical Engineering Institute, St. Peterburg, Russia, in 1964, PhD and DSc degrees from the Slovak University of Technology, Bratislava, Slovakia, in 1971 and 1985, respectively. Since 1964 he has been with the Department of Automatic Control Systems, STU FEI in Bratislava. Since 1986 he has been a full professor. His research interests include the areas of power system control, decentralized control of large-scale systems, robust control, predictive control and optimization. He is author or coauthor of more than 300 scientific papers.

Ladislav Körösi was born in Šal'a, Slovakia in 1977. He received his MSc and $\mathrm{PhD}$ degrees from the Slovak University of Technology, Bratislava in 2002 and 2010. Since 2005 he works at the Institute of Robotics and Cybernetics, STU FEI in Bratislava. His research interests include the areas of neural networks, fuzzy systems, genetic algorithms and control systems. He is author or coauthor of more than 50 scientific papers. 